\title{
COMPARISON OF WHO AND RECIST RESPONSE CRITERIA IN LOCALLY ADVANCED BREAST CANCER- A DESCRIPTIVE STUDY
}

\author{
Vishnu Kumar Sundararaju ${ }^{1}$ \\ 1 Junior Resident, Department of Surgery, JIPMER.
}

\begin{abstract}
BACKGROUND

The current trend in management of Locally Advanced Breast Cancer (LABC) has changed from radical surgery to multimodality approach involving surgery, radiotherapy and chemotherapy. The efficacy of anticancer drugs is assessed by objective tumour response. The standard WHO response criteria have been used traditionally, however, with improvement in imaging modalities, new guidelines using unidimensional measurement has been proposed by the Response Evaluation Criteria in Solid Tumours [RECIST] group. Our study was aimed to compare the standard WHO response criteria with the new RECIST criteria in Locally Advanced Breast Cancer [LABC].
\end{abstract}

\section{MATERIALS AND METHODS}

Data were collected from 52 patients with locally advanced breast cancer to compare the two response assessment methods. All patients were biopsy-proven infiltrating ductal carcinoma cases and were evaluated completely including metastatic workup. Clinical measurement of the tumour was done using callipers. Contrast enhanced CT scan of thorax was done to assess tumour size and nodal status. Three cycles of chemotherapy [Paclitaxel and Adriamycin] was given at 21 days interval. Post-chemotherapy CECT scan was done two weeks after the third cycle of chemotherapy and the tumour size was assessed. Clinical measurement of the tumour was also done post chemotherapy. WHO and RECIST response criteria were assessed in all patients and the results were compared using kappa $\mathrm{K}$ statistic to test concordance for overall response rate.

\section{RESULTS}

WHO criteria showed complete response in 10/52, partial response in 13/52 and 29/52 had no response. According to RECIST criteria, 8/52 had complete response, 20/52 had a partial response and 24/52 had no response. The response rate according to WHO criteria was $44.2 \%$. With RECIST criteria the response rate was $53.8 \%$. Seven patients were reclassified. There was good agreement between the unidimensional and bidimensional criteria in 22 of 23 responses (95.7\%). The kappa $\mathrm{K}$ statistic for concordance for overall response was 0.733 .

\section{CONCLUSION}

It is concluded from our study that the new RECIST criteria is comparable to the standard WHO criteria in evaluating locally advanced breast cancer. The RECIST criteria is a simpler measurement tool which can be used in daily practice and also gives a more accurate way of assessment about tumour response.

\section{KEYWORDS}

Locally Advanced Breast Cancer, WHO Criteria, RECIST Criteria.

HOW TO CITE THIS ARTICLE: Sundararaju VK. Comparison of WHO and RECIST response criteria in locally advanced breast cancer- a descriptive study. J. Evolution Med. Dent. Sci. 2017;6(65):4695-4698, DOI: 10.14260/Jemds/2017/1016

\section{BACKGROUND}

Breast cancer is becoming the number one cancer in females pushing carcinoma cervix to the second spot.[1] The current trend in management of Locally Advanced Breast Cancer (LABC) has changed from radical surgery to multimodality approach involving surgery, radiotherapy and chemotherapy. Locally advanced breast cancer is generally defined as bulky primary tumour and/or extensive adenopathy.

This includes patients with $\mathrm{T} 3(>5 \mathrm{~cm})$ or T4 tumours (chest wall fixation or skin ulceration and/or satellitosis) and N2/N3 disease (matted axillary and/or internal mammary metastasis). Locally advanced disease also includes patients

Financial or Other, Competing Interest: None.

Submission 25-07-2017, Peer Review 06-08-2017,

Acceptance 08-08-2017, Published 14-08-2017.

Corresponding Author:

Dr. Vishnu Kumar,

Junior Resident,

Department of Surgery, JIPMER.

E-mail: vichu.doc08@gmail.com

DOI: $10.14260 /$ jemds/2017/1016

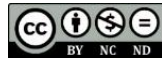

with fixed axillary lymph nodes or ipsilateral supraclavicular, infraclavicular, or internal mammary nodal involvement. Thus, all of stage III disease is considered locally advanced, as is a subset of stage IIB (T3N0).[2,3]

Neoadjuvant chemotherapy [NACT] also known as primary systemic therapy or induction therapy has become an integral part in the management of LABC.[4,5] Many inoperable tumours reduce in size after chemotherapy (downstaging) making the tumour operable. The response to neoadjuvant chemotherapy influences surgical management and also facilitates to plan about the further adjuvant chemotherapy. The reduction in tumour size also makes breast conservation surgery feasible in certain cases. All the tumours do not respond in the same manner. Response is assessed by tumour size measurement before and after chemotherapy.[6]

In early 1980s, the World Health Organization (WHO) developed recommendations to standardise the response assessment. WHO response criteria, bidimensional measurement [Table 1] is used as a standard method for evaluation of tumour response.[7] Complete response implies disappearance of all measurable or evaluable disease, signs, 
symptoms, and biochemical changes related to the tumour for at least 4 weeks, during which time no new lesions may appear. Partial response implies a reduction of greater than $50 \%$ in the sum of the products of the perpendicular diameters of all measurable lesions (Compared with pretreatment measurements) lasting at least 4 weeks. During this period no new lesions may appear and no existing lesion may enlarge. Stable disease is a less than $50 \%$ reduction or a less than $25 \%$ increase in the sum of the products of the two perpendicular diameters of all measured lesions, and the appearance of no new lesions for 8 weeks. Progression or relapse is defined as an increase in the product of two perpendicular diameters of any measurable lesion by greater than $25 \%$ over the size present at entry into the study or, for patients who respond, over the size at time of maximum regression, or the appearance of new areas of malignant disease. Errors may occur in measuring and calculating the sum and products of the two dimensions which may affect the treatment plan of the patient.

With advancement in technology and newer imaging modalities, a newer criteria for evaluation of response was developed in 1998 by the RECIST group [Table 2] which was an unidimensional measurement (the sum of maximum diameters of the tumours).[8] RECIST criteria offers a simplified, conservative, extraction of imaging data. They presume that linear measures are an adequate substitute for 2-D methods and hence four response categories are registered. Complete response(CR) indicates disappearance of all target lesions; Partial response (PR) denotes at least $30 \%$ decrease in the sum of the longest diameters (LD) of target lesions, taking reference the baseline sum LD. Progressive disease (PD) denotes at least 20\% increase in the sum of the longest diameters of the target lesion, taking as reference the smallest sum LD recorded since the treatment started or the appearance of one or more new lesions; and Stable disease (SD) indicates neither sufficient shrinkage to qualify for PR nor sufficient increase to qualify for PD, taking as reference the smallest sum LD since the treatment started.

With this background, a descriptive study was aimed to determine the correlation between the WHO response criteria and RECIST response criteria in Locally Advanced Breast Cancer following Neoadjuvant chemotherapy.

\section{MATERIALS AND METHODS}

Fifty-two patients with Locally Advanced Breast Cancer according to the AJCC (American Joint Committee on Cancer) classification were included in the study after obtaining informed consent. All patients had a tumour size of $5 \mathrm{~cm}$ or more and also had clinically palpable lymph nodes. Core needle biopsy of the tumour was taken under local anaesthesia and aseptic precautions with $18 \mathrm{G}$ Bard Trucut biopsy needle. The specimens were sent in formalin to confirm the pathological diagnosis in all patients. The tumour type, SBR grade,[9] oestrogen receptor, progesterone receptor and HER2/neu receptor were assessed. Routine investigations included complete blood counts, renal function tests and liver function tests. Ultrasound abdomen was done to look for liver metastasis. Echocardiography was done to assess left ventricular function as all patients received Adriamycin based chemotherapy.
Detailed history was taken and clinical assessment of the tumour was done. The measurement of the two longest diameters of the tumour was done using callipers. Contrast enhanced CT scan of thorax was done in all patients after ruling out any allergic reaction to the contrast. Thus, the tumour size and axillary lymph nodal status were determined both clinically and by using Contrast enhanced CT of thorax before chemotherapy.

All the patients were subjected to three cycles of Paclitaxel and Adriamycin based chemotherapy at twentyone days interval. Complete blood counts were done prior to each cycle to check for bone marrow suppression. The response to chemotherapy was assessed again at two weeks after the completion of the third cycle of chemotherapy. Clinical examination was done and the tumour was again measured using callipers and noted. Contrast enhanced CT of thorax was done and the tumour measurements were noted. Based on the pre and post-chemotherapy measurements, the response was assessed as per WHO and RECIST criteria respectively [Table.3]. The results were compared using kappa statistic to test concordance for the overall response rate.

\section{RESULTS}

Fifty-two patients were recruited for the study. Both prechemotherapy and post-chemotherapy CECT scans of thorax were taken for 52 patients and the response was assessed as per WHO and RECIST criteria.

According to WHO criteria, out of 52 patients there were 23 responders and 29 non-responders. Of the 23/52 responders, 10 had complete response, 13 had a partial response. Non-responders were $29 / 52$. The overall response rate according to WHO criteria was $44.2 \%$.

According to RECIST criteria, out of 52 patients there were 28 responders and 24 non-responders. Of the 28/52 responders, 8 had complete response, 20 had a partial response. Non-responders were 24/52. The overall response rate according to RECIST criteria was $53.8 \%$.

Seven patients were reclassified. One patient showed partial response in WHO criteria but no response according to RECIST criteria. Six patients had static disease in WHO criteria but showed partial response in RECIST criteria.

Kappa statistic was used to test concordance for overall response rate between the two criteria. There was good agreement between the two criteria in 22 of 23 responses (95.7\%) and the kappa statistic for concordance in overall response was 0.733 .

\begin{tabular}{|c|c|}
\hline Methods & $\begin{array}{c}\text { Sum of Products of Two Longest } \\
\text { Diameters in Perpendicular } \\
\text { Dimensions (Bidimensional) }\end{array}$ \\
\hline $\begin{array}{c}\text { Complete response } \\
\text { (CR) }\end{array}$ & $\begin{array}{c}\text { Disappearance of all known lesion(s); } \\
\text { confirmed at 4 weeks }\end{array}$ \\
\hline Partial response (PR) & $\geq 50 \%$ decrease; confirmed at 4 weeks \\
\hline Stable disease (SD) & Neither PR nor PD criteria met \\
\hline $\begin{array}{c}\text { Progressive disease } \\
\text { (PD) }\end{array}$ & $\begin{array}{c}\geq 25 \% \text { increase; no CR, PR or SD } \\
\text { documented before increased disease, } \\
\text { or new lesion(s) }\end{array}$ \\
\hline \multicolumn{2}{|c|}{ Table 1. WHO Criteria } \\
\hline
\end{tabular}




\begin{tabular}{|c|c|}
\hline Methods & $\begin{array}{c}\text { Sum of Longest Diameters } \\
\text { (Unidimensional) }\end{array}$ \\
\hline Complete response (CR) & $\begin{array}{c}\text { Disappearance of all known } \\
\text { lesion(s); confirmed at 4 weeks }\end{array}$ \\
\hline Partial response (PR) & $\begin{array}{c}\geq 30 \% \text { decrease; confirmed at 4 } \\
\text { weeks }\end{array}$ \\
\hline Stable disease (SD) & Neither PR nor PD criteria met \\
\hline Progressive disease (PD) & $\begin{array}{c}\geq 20 \% \text { increase; no CR, PR or SD } \\
\text { documented before increased } \\
\text { disease, or new lesion(s) }\end{array}$ \\
\hline \multicolumn{2}{|c|}{ Table 2. RECIST Criteria } \\
\hline \multicolumn{2}{|c}{}
\end{tabular}

\begin{tabular}{|c|c|c|c|c|}
\hline \multicolumn{2}{|c|}{} & \multicolumn{2}{c|}{ WHO } & \multirow{2}{*}{ Total } \\
\cline { 3 - 5 } & No Response & Response & \\
\hline \multirow{3}{*}{ RECIST } & $\begin{array}{c}\text { No } \\
\text { response }\end{array}$ & 23 & 1 & $\begin{array}{c}24 \\
(46.2 \%)\end{array}$ \\
\cline { 2 - 5 } & Response & 6 & 22 & $\begin{array}{c}28 \\
(53.8 \%)\end{array}$ \\
\hline \multirow{3}{*}{} & Total & $\begin{array}{c}\mathbf{2 9} \\
(\mathbf{5 5 . 8 \% )}\end{array}$ & $\begin{array}{c}\mathbf{2 3} \\
\mathbf{( 4 4 . 2 \% )}\end{array}$ & $\begin{array}{c}\mathbf{5 2} \\
(\mathbf{1 0 0} \%)\end{array}$ \\
\hline
\end{tabular}

Table 3. Correlation between WHO and RECIST Response

Kappa $=0.733$.

\section{DISCUSSION}

The current management of locally advanced breast cancer includes chemotherapy and radiotherapy besides surgical treatment. Neoadjuvant chemotherapy has become an integral part in the management of locally advanced breast cancer. With the advent of newer anticancer drugs, the chemotherapy regimens are evolving. The efficacy of anticancer drugs is evaluated by objective tumour response. Based on this response, further treatment is planned. The method used to assess response is changing over the years.[6,8] Previously comparison of physical examination, mammography and sonography in the assessment of tumour response in 100 patients was done and it was found that physical examination was the best non-invasive predictor for assessment of tumour size and the accuracy increased when combined with sonography.[10]

However, studies done by Cocconi et al revealed that physical examination overestimated the tumour size in $23 \%$ and underestimated the response in $9 \%$ of their patients.[11] Radiological investigations available for breast cancer response evaluation are mammography, sonography, CT scan, MRI, mammoscintigraphy and PET scan. Of these mammography and sonography are the most commonly used and easily available. There are other reports which state that MRI was more reliable for assessing response to chemotherapy when compared with physical examination, mammography and sonography.[12]

Retrospective analysis of 189 breast cancer patients concluded that physical examination, sonography and mammography did not accurately correlate with residual pathologic tumour size and hence were only moderately useful for predicting response after neoadjuvant chemotherapy.[13] Akashi-Tanaka et al in their study concluded that CECT is the most accurate noninvasive technique for classifying (diffuse and localised types) and also in identifying the extent of the residual tumour after neoadjuvant chemotherapy when cases of inflammatory breast carcinoma and invasive lobular carcinoma were excluded.[14]

Investigators from Strasbourg reported on the use of Helical CT scanning to assess response to neoadjuvant chemotherapy. Fifty women with stage II or III breast cancer underwent helical CT scanning pre and post chemotherapy for primary breast cancer. Helical CT measurement of residual tumour had better correlation with residual disease by pathological assessment also than did either clinical examination or mammography. They concluded that breast helical CT can be very useful in assessing response to neoadjuvant chemotherapy.[15]

Jo Park et al compared the WHO response criteria with the RECIST guidelines. Among the 79 patients enrolled, the overall response rate according to the WHO criteria was $31.6 \%$. Using the RECIST criteria, nine patients had to be reclassified and the overall response rate was $30.4 \%$. They concluded that though both the criteria are comparable; RECIST is simple and reproducible in the measurement of response in daily practice.[16] Therasse et al in their retrospective comparison of six trials involving 795 patients found that the switch from WHO to RECIST criteria resulted in a more favourable classification. ${ }^{[8]}$

George J et al used both WHO and RECIST criteria for response assessment after neoadjuvant chemotherapy in their study of single nucleotide polymorphism in MDR 1 gene in breast cancer. The objective response by WHO criteria was $63 \%$ and with RECIST criteria was 50.6\%.[17] Sivasanker et al used RECIST criteria for assessing the response in breast cancer comparing cyclophosphamide, Adriamycin and 5fluorouracil [CAF] regime with Paclitaxel and Adriamycin and found it to be a simpler way for assessing tumour response following chemotherapy.[18]

In our study, the overall response rate according to WHO criteria was $44.2 \%$ and with RECIST criteria was $53.8 \%$. Analysis with kappa statistic showed good agreement between the unidimensional and bidimensional measurements with a concordance rate of 0.733. Erasmus et al noted interobserver and intraobserver bias in the measurement values of tumours.[19]

As the tumour measurement plays an important role in staging the disease, we need clear protocols and guidelines for the tumour measurements to remove the bias and to give the patients a better line of management by which they will be benefitted.

Although many retrospective studies have been done comparing WHO and RECIST response criteria in various solid tumours; our study was done in locally advanced breast cancer alone, which strengthens the outcome.

\section{CONCLUSION}

Our study proves that RECIST criteria is comparable to WHO criteria and is a simpler tool to assess the response. Further prospective studies in individual solid tumours will be needed to prove the reliability of RECIST criteria and to design a standard protocol for tumour measurement.

\section{ACKNOWLEDGEMENTS}

I would like to thank Dr. Kadambari and Dr. Srinivasan for their constant guidance and immense support during the entire period of study. 


\section{REFERENCES}

[1] Breast cancer in India rising rapidly. http://www.medindia.net/ news/view_news_main.asp 2010.

[2] Singletary SE, Allred C, Ashley P, et al. Revision of the American joint committee on cancer staging system for breast cancer. J Clin Oncol 2002;20(17):3628-36.

[3] Lee MC, Newman LA. Management of patients with locally advanced breast cancer. Surg Clin North America 2007;87:379-98.

[4] Waljee JF, Newman LA. Neoadjuvant systemic therapy and the surgical management of breast cancer. Surg Clin N Am 2007;87(2):399-415.

[5] Smith IC, Heys SD, Hutcheon AW, et al. Neoadjuvant chemotherapy in breast cancer: significantly enhanced response with docetaxel. Journal of Clinical Oncology 2002;20(6):1456-66.

[6] Therasse P. Measuring the clinical response. What does it mean? Eur J Cancer 2002;38(14):1817-23.

[7] Miller AB, Hoogstraten B, Staquet M, et al. Reporting results of cancer treatment. Cancer 1981;47(1):20714.

[8] Therasse P, Arbuck SG, Eisenhauer EA, et al. New guidelines to evaluate the response to treatment in solid tumors. European organization for research and treatment of cancer, national cancer institute of the United States, national cancer institute of Canada. J Natl Cancer Inst 2000;92(3):205-16.

[9] Bloom HJ, Richardson WW. Histological grading and prognosis in breast cancer. A study of 1409 cases of which 359 have been followed for 15 years. $\mathrm{Br}$ J Cancer 1957;11(3):359-77.

[10] Herrada J, Iyer RB, Atkinson EN, et al. Relative value of physical examination, mammography and breast sonography in evaluating the size of the primary tumor and regional lymph node metastases in women receiving neoadjuvant chemotherapy for locally advanced breast carcinoma. Clin Cancer Res 1997;3(9):1565-9.
[11] Cocconi G, Dblasio B, Alberti G, et al. Problems in evaluating response of primary breast cancer to systemic therapy. Breast cancer Res Treat 1984;4(4):309-13.

[12] Balu-Maestro C, Chapellier C, Bleuse A, et al. Imaging in evaluation of response to neoadjuvant breast cancer treatment benefits of MRI. Breast Cancer Res Treat 2002;72(2):145-52.

[13] Chagpar AB, Middleton LP, Sahin AA, et al. Accuracy of physical examination, ultrasonography, and mammography in predicting residual pathologic tumor size in patients treated with neoadjuvant chemotherapy. Ann Surg 2006;243(2):257-64.

[14] Akashi-Tanaka S, Fukutomi T, Watanabe T, et al. Accuracy of Contrast enhanced computed tomography in the prediction of residual breast cancer after neoadjuvant chemotherapy. Int J Cancer 2001;96(1):66-73.

[15] Moyses B, Haegele P, Rodier JF, et al. Assessment of response by breast helical computed tomography to neoadjuvant chemotherapy in large inflammatory breast cancer. Clin Breast Cancer 2002;2(4):304-10.

[16] Park JO, Lee SI, Song SY, et al. Measuring response in solid tumors: comparison of RECIST and WHO response criteria. Jpn J Clin Oncol 2003;33(10):533-7.

[17] George J, Dharanipragada K, Krishnamachari S, et al. A single-nucleotide polymorphism in the MDR1 gene as a predictor of response to neoadjuvant chemotherapy in breast cancer. Clin Breast Cancer 2009;9(3):161-5.

[18] Sivasanker M, Sistla SC, Manwar SA, et al. Clinical and pathologic response following taxane based neoadjuvant chemotherapy in locally advanced breast cancer patients in a tertiary care centre in India. Indian J of Cancer 2016;53(2):220-5.

[19] Erasmus JJ, Gladish GW, Broemeling L, et al. Interobserver and intraobserver variability in measurement of non-small-cell carcinoma lung lesions: implications for assessment of tumor response. J Clin Oncol 2003;21(13):2574-82. 